\title{
PLASMA LEVELS OF CHEMERIN, LEPTIN AND PSORIASIN AS POTENTIAL MARKERS OF SUBCLINICAL ATHEROSCLEROSIS IN PSORIASIS PATIENTS
}

\section{Nevein M. Al-sheikh ${ }^{1}$, Wafaa A. Shehata ${ }^{2}$, Shaimaa A. Hassanein ${ }^{3}$ and Waleed A. Ibrahim ${ }^{4}$ \\ ${ }^{1}$ Medical Biochemistry\& Molecular Biology, ${ }^{2}$ Dermatology \& Andrology, ${ }^{3}$ Diagnostic Radiology and ${ }^{4}$ Cardiology Departments, Faculty of Medicine, Menoufia University, Egypt.}

\section{Received 1/4/2018 - Accepted 29/11/2018}

\begin{abstract}
Chemerin, leptin and psoriasin are pro-inflammatory and immunemodulatory proteins associated with psoriasis and displayed higher circulating levels. Their relation to atherosclerosis in psoriatic patients has been investigated in numerous studies with wide-ranging results. Therefore, the present study aimed to assess plasma levels of chemerin, leptin and psoriasin and evaluate their relationship with carotid intima-media thickness (CIMT) and epicardial fat thickness (EFT) as potential predictors for subclinical atherosclerosis in psoriasis patients. The study included fifty psoriatic patients and forty age and gender matched healthy controls. Clinical severity of psoriasis was evaluated by Psoriasis Area and Severity Index (PASI). Fasting blood glucose and lipid profile were estimated. Plasma levels of high sensitivity-CRP (hs-CRP), chemerin, leptin and psoriasin were measured by ELISA. CIMT and EFT were assessed by Ultrasonography and Echocardiography, respectively. Plasma levels of hs-CRP, chemerin, leptin and psoriasin as well as CIMT and EFT were significantly elevated in psoriasis patients compared to controls. CIMT and EFT were significantly positively correlated with PASI, plasma hs-CRP, chemerin, leptin and psoriasin. Moreover, significant positive correlation was demonstrated between PASI and plasma hsCRP, chemerin, leptin and psoriasin. Multiple linear regression analyses showed that chemerin, leptin and psoriasin were
\end{abstract}

*Correspandence Aauthor (drnoua_vid@yahoo.com) 
independently correlated with CIMT and EFT and exhibited high significance for predicting their values. It can be concluded that chemerin, leptin and psoriasin might represent an important link between psoriasis and atherosclerosis. Measurements of plasma chemerin, leptin and psoriasin along with CIMT and EFT seem to be valuable potential markers of subclinical atherosclerosis in patients with psoriasis.

Key Words: Chemerin, Leptin, Psoriasin, Psoriasis and Atherosclerosis.

\section{INTRODUCTION}

Psoriasis is a T-cell mediated, chronic inflammatory skin disease characterized by hyperproliferation of keratinocytes. It is recognized by the presence of erythematous, silvery scaly and well-defined plaques particularly over extremities and scalp (Hawkes et al., 2017). It is the most predominant chronic inflammatory disorder in humans, affecting about $2-4 \%$ of the population worldwide (Michalek et al., 2017).

Psoriasis is currently prescribed as a multi-systemic inflammatory disorder rather than a disease of the skin and joints only. Atherosclerosis is one of the systemic involvements of psoriasis, where the inflammatory processes provide a major link between the two diseases. Persistent skin inflammation triggers a cascade of systemic inflammatory events resulting in premature atherosclerosis and increased risk of cardiovascular disease (CVD) in psoriasis patients (Dattilo et al., 2018). Accordingly, psoriasis seems to be an independent risk factor for subclinical atherosclerosis and CVD even after adjusting for conventional risk factors such as smoking, hypertension, diabetes and obesity (Kolliker Frers et al., 2018).

Chemerin is a novel adipokine mainly expressed in adipocytes of white adipose tissue. It is a chemo-attractant protein with inflammatory and immune-modulatory functions. It plays an essential role in cell differentiation, angiogenesis, lipid metabolism and insulinmediated pathways (Eichelmann et al., 2017). High circulating chemerin level and up-regulation of its gene were detected in a variety of chronic inflammatory conditions. It is highly expressed in psoriatic lesions, thus indicates its involvement in the disease pathogenesis by 
stimulating skin innate immunity and inducing chemotaxis (Banas et al., 2015).

Leptin is one of the classical adipokines that secreted mainly by adipose tissue. It regulates several metabolic pathways such as; appetite and body weight, glucose and lipid homeostasis and insulin sensitivity (Zhang and Chua, 2017). It controls many cellular inflammatory cascades through production of pro-inflammatory cytokines, modulating T-helper cell activity and promoting keratinocyte proliferation, therefore its definite contribution in the pathogenesis of psoriasis (Kyriakou et al., 2017).

Psoriasin (hS100A7) is a member of the multigenic S100 calciumbinding proteins family, expressed mainly by epithelial cells. Because of its role in calcium binding and signaling, it has involved in cellular proliferation, differentiation, apoptosis, inflammatory and immunemodulatory activity (Cunden et al., 2017). It was first recognized as an over-expressed protein in psoriatic lesions acting as proinflammatory mediator and chemo-attractant molecule for T-cells, hence its role in the disease pathogenesis (Ekman et al., 2017).

Carotid intima-media thickness (CIMT) is a non-invasive imaging tool, used to diagnose atherosclerotic process at its pre-occlusive stage i.e. subclinical atherosclerosis in high-risk individuals. Intimal smooth muscle proliferation and atherogenic aggregation may explain the increased CIMT and early-onset atherosclerosis in psoriasis patients (Santilli et al., 2016). Epicardial fat thickness (EFT) is a metabolically active tissue that refers to cardiac adiposity and produces various atherogenic and inflammatory cytokines. It contributes to cellular infiltration, angiogenesis and endothelial dysfunction of the vascular intima, and consequently the development of atherosclerosis (Wang et al., 2016). Therefore, our study aimed to measure the plasma levels of chemerin, leptin and psoriasin and evaluate their possible relationship with CIMT and EFT as potential predictors for subclinical atherosclerosis in psoriasis patients.

\section{Subjects:}

\section{MATERIALS AND METHODS}

This study was conducted at Medical Biochemistry, Dermatology, Diagnostic Radiology and Cardiology Departments, Faculty of Medicine, Menoufia University, Egypt, during the period from March to December 2017. A total number of (90) subjects were enrolled in 
the study and classified into two groups. Group (1): included (50) patients with chronic plaque psoriasis (their ages ranged from 36-54 years), were recruited from dermatology outpatient clinic. Group (2): included (40) age- and gender-matched healthy controls (their ages ranged from 37-52 years), with negative family history of psoriasis. Written consents were obtained from all subjects after approval of the study protocol by the Human Rights in Research Committee at Menoufia University.

Psoriasis patients were clinically assessed using Psoriasis Area and Severity Index (PASI) Score. Both intensity and extent of psoriatic plaques were calculated for 4 anatomical regions (head, arms, trunk and legs) by a physician who specified: (i) the percent of area of skin involved and (ii) the severity of three clinical signs; erythema, induration and desquamation on a scale from 0 to 4 indicating; no involvement, slight, moderate, severe and very severe, respectively. The patients were categorized according to their PASI score as mild $(<10)$; moderate (10-14) and severe psoriasis (>14) (Kothiwala et al., 2016). All subjects of the study were submitted to complete history taking, overall clinical examinations, blood pressure assessment and anthropometric measurements. Body mass index (BMI) was calculated as [wt $(\mathrm{kg}) /$ height $\left.(\mathrm{m})^{2}\right]$ (Janssen, 2013). For Waist/ Hip Ratio (WHR), waist circumference was measured between the lowest rib and iliac crest and hip circumference was taken at the widest area of the hips at the greatest protuberance of the buttocks. The waist measurement was then divided by the hip measurement (Rashiti et al., 2017). Exclusion criteria were: patients $<18$ or $>60$ years; smoking; BMI $>35 \mathrm{~kg} / \mathrm{m}^{2}$; history of CVD, DM, hypertension, dyslipidemia; pregnant and lactating females and the use of retinoid therapy 6 months prior to study enrolment.

\section{Blood Sampling:}

After 12 hours of an overnight fasting, $10 \mathrm{ml}$ of venous blood were collected from all subjects and distributed as follow: One ml into sodium flouride tube for blood glucose estimation; $5 \mathrm{ml}$ into EDTA tube and $4 \mathrm{ml}$ into a plain tube allowed to clot at room temperature. Both EDTA and plain tubes were centrifuged at 4000 r.p.m for 10 minutes, and the supernatant serum and plasma were separated and stored at $-80^{\circ} \mathrm{C}$ until further analysis. 
Laboratory and Radiological Investigations: All subjects were submitted to the following:

-Fasting blood glucose was measured based on enzymatic colorimetric method, using (Spinreact kit, SPAIN) (Trinder, 1969).

-Fasting lipid profile was measured based on colorimetric method, using (Cell Biolabs kit, San Diego, CA, USA). Total cholesterol (Rifai and Warnick, 2006); triglycerides (Fossati and Prenciphe, 1982); HDL-c (Gordon et al., 1977) and LDL-c was calculated according to Friedewald formula (Friedewalde et al., 1972).

-Plasma high sensitivity- CRP was measured using (hs-CRP ELISA Kit, My BioSource Int, San Diego, USA).The detection range for the assay was $0.25-8 \mathrm{mg} / \mathrm{L}$ (Yang et al., 2017).

-Plasma Chemerin was measured using (Chemerin ELISA Kit, BioVendor Laboratorni, AS, Czech Republic).The detection range for the assay was 0.25-8 ng/mL (Aksu et al., 2017).

-Plasma Leptin was measured using (Human Leptin ELISA Kit, BioVendor Laboratorni, AS, Czech Republic). The detection range for the assay was 1-50 ng/mL (Asha et al., 2014).

-Plasma Psoriasin was measured using (Psoriasin ELISA Kit, CircuLex, Nagano, Japan). The detection range for the assay was $0.12-90 \mathrm{ng} / \mathrm{mL}$ (Awad et al., 2018).

-Carotid Intima-Media Thickness (CIMT) was measured using BMode Ultrasonography (Aplio 400 Ultrasound, Toshiba, Japan). It was performed bilaterally, $1 \mathrm{~cm}$ proximal to the carotid bulb in a longitudinal view and calculated as the arithmetic mean of right and left carotid arteries measurements. CIMT value of $1.0 \mathrm{~mm}$ was considered as cut-off point for a significant intima-media thickness, predicting subclinical atherosclerosis (Awad et al., 2018).

-Epicardial Fat Thickness (EFT) was measured using Transthoracic Echocardiography (Vivid 9, General Electric, Norway). It was calculated as the mean value of the measurements at end-diastole in three cardiac cycles. EFT value of $6.3 \mathrm{~mm}$ was taken as a cut-off point, indicating subclinical atherosclerosis (Cetin et al., 2013).

\section{Statistical Analysis:}

Data were statistically analyzed using SPSS version 20 (SPSS Inc., Chicago, Illinois, USA). Quantitative data were expressed as mean \pm standard deviation and qualitative data were expressed as percentage. Normally distributed quantitative data were tested using independent 
t-test. Chi square test $(\chi 2)$ was used to verify the association between qualitative variables. Pearson's correlation coefficients (r) were calculated to assess the relation of PASI Score, CIMT and EFT with other studied risk variables. A Multiple linear regression analysis was constructed to evaluate the impact of independent variables (predictors) on dependant variables (CIMT \& EFT). P-values at $<0.05$ were considered statistically significant.

\section{RESULTS}

The present study included; 50 patients with psoriasis as Group (1), they were 23 males and 27 females with mean age of $45.3 \pm 6.5$ years; and 40 age and gender matched healthy controls as Group (2), they were 20 males and 20 females with mean age of $44.5 \pm 5.9$ years. No significant differences were demonstrated between psoriasis patients and controls regarding; age, gender, BMI, SBP, DBP or FBG $(p>0.05)$. High statistically significant differences were identified between both groups as regard; WHR, TC, TGs, HDL-c, LDL-c and hs-CRP ( $p<0.001)$. Plasma chemerin, leptin and psoriasin were significantly higher in psoriasis patients than in controls; $(351.2 \pm 68.2$ vs. $210.6 \pm 46.5),(41.4 \pm 7.5$ vs. $11.4 \pm 3.2)$ and $(56.3 \pm 10.5$ vs. $22.6 \pm$ $5.4)$, respectively $(\mathrm{p}<0.001)$. CIMT and EFT were markedly elevated in psoriasis patients than in controls; $(1.06 \pm 0.31$ vs. $0.62 \pm 0.08),(6.8 \pm$ 1.12 vs. $3.2 \pm 0.52)$, respectively $(\mathrm{p}<0.001)$ [Table 1 ].

Pearson correlation analysis revealed that; PASI Score was positively correlated with WHR, TC, TGs, LDL-c, hs-CRP, chemerin, leptin, psoriasin, CIMT and EFT, while inversely correlated with HDL-c $(p<0.001)$. However, PASI did not show any correlation with age, gender, BMI, SBP, DBP or FBG ( $p>0.05$ ) [Table 2]. CIMT and EFT showed significant positive correlations with WHR, TC, TGs, LDL-c, hs-CRP, chemerin, leptin and psoriasin and significant inverse correlation with HDL-c $(\mathrm{p}<0.001)$. However, CIMT and EFT did not correlate with age, gender, BMI, SBP, DBP or FBG ( $p>0.05)$ [Table 3]. Multiple linear regression analysis with CIMT as a dependent variable showed that; hs-CRP, chemerin, leptin and psoriasin were independently correlated with CIMT. The highest significant predictor for CIMT was chemerin $(\beta=0.004, \mathrm{~T}=3.24, \mathrm{P}=0.002)$, followed by psoriasin $(\beta=0.029, \mathrm{~T}=3.05, \mathrm{P}=0.004)$, then hs-CRP $(\beta=0.16, \mathrm{~T}=2.55$, $\mathrm{P}=0.01)$ and leptin $(\beta=0.46, \mathrm{~T}=2.11, \mathrm{P}=0.04)$ [Table 4]. Multiple 
linear regression with EFT as a dependent variable showed that; chemerin, leptin and psoriasin were independently correlated with EFT. The highest significant predictor for EFT was leptin $(\beta=0.21$, $\mathrm{T}=3.41, \mathrm{P}=0.001)$, then chemerin $(\beta=0.38, \mathrm{~T}=3.24, \mathrm{P}=0.001)$ and psoriasin $(\beta=0.26, \mathrm{~T}=2.88, \mathrm{P}=0.002)$ [Table 5].

Table (1): Clinical, Laboratory and Radiological Variables of the Studied Groups.

\begin{tabular}{|c|c|c|c|c|}
\hline & $\begin{array}{c}\text { Psoriasis } \\
\text { Group } \\
\text { G } 1(\mathrm{~N}=\mathbf{5 0})\end{array}$ & $\begin{array}{c}\text { Control Group } \\
\text { G } 2(\mathrm{~N}=40)\end{array}$ & T Test & P Value \\
\hline $\begin{array}{l}\text { Gender, N (\%): } \\
\text { Males } \\
\text { Females }\end{array}$ & $\begin{array}{l}23(46 \%) \\
27(54 \%)\end{array}$ & $\begin{array}{l}20(50 \%) \\
20(50 \%)\end{array}$ & $\chi^{2}=0.14$ & 0.71 \\
\hline Age (years) & $45.3 \pm 6.5$ & $44.5 \pm 5.9$ & 0.6 & 0.54 \\
\hline BMI $\left(\mathrm{kg} / \mathrm{m}^{2}\right)$ & $28.5 \pm 2.2$ & $27.7 \pm 1.9$ & 1.8 & 0.07 \\
\hline WHR & $0.98 \pm 0.05$ & $0.89 \pm 0.02$ & 10.7 & $<0.001^{*}$ \\
\hline SBP(mmHg) & $122 \pm 3.2$ & $120.7 \pm 3.4$ & 1.86 & 0.06 \\
\hline DBP(mmHg) & $79 \pm 1.7$ & $78.4 \pm 1.6$ & 1.7 & 0.09 \\
\hline PASI Score & $16.4 \pm 4.2$ & --------------------- & ------------ & -------------- \\
\hline $\begin{array}{l}\text { Disease Duration } \\
\text { (months) }\end{array}$ & $47.5 \pm 6.5$ & --------------------- & ------------- & --------------- \\
\hline FBG (mg/dl) & $94 \pm 7.5$ & $91.3 \pm 6.7$ & 1.78 & 0.08 \\
\hline TC (mg/dl) & $257.4 \pm 22.7$ & $165.5 \pm 17.4$ & 21.1 & $<0.001^{*}$ \\
\hline TGs (mg/dl) & $205.3 \pm 21.6$ & $129.2 \pm 12.5$ & 19.8 & $<0.001^{*}$ \\
\hline HDL-c (mg/dl) & $32.5 \pm 3.7$ & $44.7 \pm 4.7$ & 13.8 & $<0.001^{*}$ \\
\hline LDL-c (mg/dl) & $183.8 \pm 14.7$ & $94.8 \pm 10.2$ & 32.5 & $<0.001^{*}$ \\
\hline hs-CRP (mg/L) & $4.59 \pm 1.12$ & $0.98 \pm 0.29$ & 19.8 & $<0.001^{*}$ \\
\hline Chemerin(ng/ml) & $351.2 \pm 68.2$ & $210.6 \pm 46.5$ & 11.1 & $<0.001^{*}$ \\
\hline Leptin(ng/ml) & $41.4 \pm 7.5$ & $11.4 \pm 3.2$ & 23.6 & $<0.001^{*}$ \\
\hline Psoriasin (ng/ml) & $56.3 \pm 10.5$ & $22.6 \pm 5.4$ & 18.4 & $<0^{0.001}{ }^{*}$ \\
\hline Mean CIMT (mm) & $1.06 \pm 0.31$ & $0.62 \pm 0.08$ & 8.7 & $<0^{0.001}{ }^{*}$ \\
\hline EFT(mm) & $6.8 \pm 1.12$ & $3.2 \pm 0.52$ & 18.8 & $<0.001^{*}$ \\
\hline
\end{tabular}

*Significant value $=p<0.05$ (obtained through $\chi^{2}=$ chi square test and $\mathrm{T}=$ independent t-test). Data are presented as Mean \pm SD. BMI: Body mass index; WHR: Waist hip ratio; SBP: Systolic blood pressure; DBP: Diastolic blood pressure; PASI: Psoriasis area and severity index score; FBG: Fasting blood glucose; TC: Total cholesterol; TGs: Triglycerides; HDL-c: High-density lipoprotein cholesterol; LDL-c: Low-density lipoprotein cholesterol; hs-CRP: High sensitivity C-reactive protein; CIMT: Carotid intima-media thickness; EFT: Epicardial fat thickness. 
Table (2): Correlation Analysis of PASI Score with the Studied Variables in Psoriasis Patients.

\begin{tabular}{|l|c|c|}
\hline \multirow{2}{*}{ Variables } & \multicolumn{2}{|c|}{ PASI Score } \\
\cline { 2 - 3 } & r & P value \\
\hline WHR & 0.40 & $0.004^{*}$ \\
\hline TC (mg/dl) & 0.42 & $0.003^{*}$ \\
\hline TGs (mg/dl) & 0.72 & $<0.001^{*}$ \\
\hline HDL-c (mg/dl) & -0.68 & $<0.001^{*}$ \\
\hline LDL-c (mg/dl) & 0.41 & $0.004^{*}$ \\
\hline hs-CRP (mg/L) & 0.42 & $0.003^{*}$ \\
\hline Chemerin(ng/ml) & 0.45 & $0.001^{*}$ \\
\hline Leptin(ng/ml) & 0.74 & $<0.001^{*}$ \\
\hline Psoriasin (ng/ml) & 0.73 & $<0.001^{*}$ \\
\hline Mean CIMT (mm) & 0.76 & $<0.001^{*}$ \\
\hline EFT (mm) & 0.42 & $0.002^{*}$ \\
\hline
\end{tabular}

*Significant value $=\mathrm{p}<0.05$. r= Pearson's correlation coefficients. PASI: Psoriasis area and severity index score; WHR: Waist hip ratio; TC: Total cholesterol; TGs: Triglycerides; HDL-c: High-density lipoprotein cholesterol; LDL-c: Low-density lipoprotein cholesterol; hs-CRP: High sensitivity C-reactive protein; CIMT: Carotid intima-media thickness; EFT: Epicardial fat thickness.

Table (3): Correlation Analysis of [CIMT and EFT] with the Studied Variables in Psoriasis Patients.

\begin{tabular}{|l|c|c|c|c|}
\hline \multirow{2}{*}{ Variables } & \multicolumn{2}{c|}{ CIMT(mm) } & \multicolumn{2}{c|}{ EFT(mm) } \\
\cline { 2 - 5 } & r & P Value & r & P Value \\
\hline WHR & 0.42 & $0.002^{*}$ & 0.65 & $<0.001^{*}$ \\
\hline TC (mg/dl) & 0.44 & $0.001^{*}$ & 0.43 & $0.002^{*}$ \\
\hline TGs (mg/dl) & 0.41 & $0.004^{*}$ & 0.45 & $0.001^{*}$ \\
\hline HDL-c (mg/dl) & -0.43 & $0.002^{*}$ & -0.42 & $0.003^{*}$ \\
\hline LDL-c (mg/dl) & 0.41 & $0.003^{*}$ & 0.40 & $0.004^{*}$ \\
\hline hs-CRP (mg/L) & 0.68 & $<0.001^{*}$ & 0.45 & $0.001^{*}$ \\
\hline Chemerin(ng/ml) & 0.69 & $<0.001^{*}$ & 0.70 & $<0.001^{*}$ \\
\hline Leptin(ng/ml) & 0.74 & $<0.001^{*}$ & 0.75 & $<0.001^{*}$ \\
\hline Psoriasin (ng/ml) & 0.71 & $<0.001^{*}$ & 0.74 & $<0.001^{*}$ \\
\hline
\end{tabular}

*Significant value $=p<0.05 . \mathbf{r}=$ Pearson's correlation coefficients. CIMT: Carotid intima-media thickness; EFT: Epicardial fat thickness. WHR: Waist hip ratio; TC: Total cholesterol; TGs: Triglycerides; HDL-c: High-density lipoprotein cholesterol; LDL-c: Low-density lipoprotein cholesterol; hs-CRP: High sensitivity C-reactive protein. 
Table (4): Multiple Linear Regression Analysis with CIMT as a Dependent Variable.

\begin{tabular}{|l|c|c|c|}
\hline Independent Variables & $\boldsymbol{\beta}$ & T & P Value \\
\hline WHR & 1.12 & 0.84 & 0.41 \\
\hline PASI Score & 0.01 & 1.55 & 0.12 \\
\hline hs-CRP (mg/L) & 0.16 & 2.55 & $0.01^{*}$ \\
\hline Chemerin(ng/ml) & 0.004 & 3.24 & $0.002^{*}$ \\
\hline Leptin(ng/ml) & 0.46 & 2.11 & $0.04^{*}$ \\
\hline Psoriasin (ng/ml) & 0.029 & 3.05 & $0.004^{*}$ \\
\hline
\end{tabular}

$*$ Significant value $=\mathrm{p}<0.05$. $\boldsymbol{\beta}$ : Beta standardized coefficient. CIMT: Carotid intima-media thickness; WHR: Waist hip ratio; PASI: Psoriasis area and severity index score; hs-CRP: High sensitivity C-reactive protein.

Table (5): Multiple Linear Regression Analysis with EFT as a Dependent Variable.

\begin{tabular}{|l|c|c|c|}
\hline \multicolumn{1}{|c|}{ Independent Variables } & $\boldsymbol{\beta}$ & T & P Value \\
\hline WHR & 0.63 & 0.46 & 0.64 \\
\hline PASI Score & 0.45 & 0.75 & 0.58 \\
\hline hs-CRP (mg/L) & 0.38 & 1.51 & 0.13 \\
\hline Chemerin(ng/ml) & 0.38 & 3.24 & $0.001^{*}$ \\
\hline Leptin(ng/ml) & 0.21 & 3.41 & $0.001^{*}$ \\
\hline Psoriasin (ng/ml) & 0.26 & 2.88 & $0.002^{*}$ \\
\hline
\end{tabular}

*Significant value $=p<0.05$. $\beta$ : Beta standardized coefficient. EFT: Epicardial fat thickness.

\section{DISCUSSION}

Psoriasis is the most prevalent chronic inflammatory disease of the skin and joints and associated with serious multi-systemic consequences. Psoriasis patients are at a higher risk to develop CVD, especially atherosclerosis due to the remarkable similarity of inflammatory pathways between psoriasis and atherosclerosis. Cardiovascular diseases are the commonest cause of death in patients with severe psoriasis, thus early detection of subclinical atherosclerosis might be beneficial in reducing cardiovascular morbidity and mortality (Dattilo et al., 2018). Numerous researches 
have recently focused on novel biomarkers that could aid in psoriasis diagnosis and predicting its co-morbid effects. Chemerin, leptin and psoriasin are pro-inflammatory, immune-modulatory molecules that have displayed higher systemic circulating levels, over-expression in psoriatic skin lesions as well as striking association with the disease severity and co-morbidities (Aksu et al., 2017; Kyriakou et al., 2017; Awad et al., 2018).

Several studies have demonstrated higher CIMT in psoriasis patients and independently from atherosclerotic risk factors, which indicate the strong relation between psoriasis and carotid atherosclerosis (Dinić et al., 2016; Santilli et al., 2016). Furthermore, EFT showed greater values in psoriasis patients, being as a recent cardio-metabolic risk factor and contributing to the development of atherosclerosis (Wang et al., 2016). Therefore, this study aimed to assess the plasma levels of chemerin, leptin and psoriasin and investigated their relation with CIMT and EFT as possible predictors for subclinical atherosclerosis in psoriasis patients.

In the current study; age, gender, BMI, blood pressure and blood glucose were matched in patients and controls to exclude their probable effects in estimating the studied atherogenic parameters. However, waist/hip ratio was significantly elevated in psoriatic patients than in controls, as well as it was positively correlated with the disease severity as indicated by PASI. These findings were in agreement with the results of Asha et al. (2014) and Dattilo et al. (2018) who demonstrated a significant association between psoriasis severity and abdominal adiposity.

The relation between psoriasis and obesity is assumed bidirectional, where psoriasis predisposes patients to obesity and obesity may be a risk factor for developing psoriasis. Abdominal adiposity is an active metabolic organ producing adipokines as leptin and chemerin. The aberrant production of these adipokines promotes the chronic inflammatory cascades in psoriasis. On the other hand, systemic inflammatory process in psoriasis especially in its severe state might cause weight gain and fat mass redistribution (El-Boghdady et al., 2018).

The present study revealed, higher levels of TC, TGs, and LDL-c and lower levels of HDL-c in patients compared to controls. Additionally TC, TGs, and LDL-c were directly correlated with the 
severity of psoriasis while, HDL-c was indirectly correlated. These results came in line with Dinić et al. (2016) and Awad et al. (2018) who reported significant dyslipidemia in psoriasis patients that was strongly associated with the disease duration and severity.

Dyslipidemia is the most prevalent metabolic abnormality existing in psoriasis and correlate positively with the disease severity. Excess adiposity and pro-inflammatory activities induced by adipocytederived cytokines as leptin seem to have a crucial role in the excessive changes of lipids and lipoproteins. This inflammatory process and dyslipidemia in psoriasis especially its severe variants could explain the high risk of developing atherosclerosis (Furue et al., 2017).

In this study, hs-CRP was highly elevated in psoriasis patients versus controls and positively correlated with disease severity. Aksu et al. (2017) have obtained matching results and reported that; chronic inflammatory pathway in psoriasis can induce systemic and vascular inflammations and thrombosis with massive production of systemic inflammatory markers as CRP.

Our results demonstrated significant increases in plasma chemerin, leptin and psoriasin in psoriasis patients, as well as positive correlations with the psoriasis severity. In agreement with these findings, Banas et al. (2015) and Aksu et al. (2017) reported that chemerin was markedly increased in the blood and highly expressed in the dermis of psoriasis patients. Asha et al. (2014) and Kyriakou et al. (2017) established higher leptin level in psoriasis patients in addition to a positive correlation with PASI. Furthermore, high circulating level of psoriasin and its over-expression in the skin of psoriasis patients were found by Ekman et al. (2017) and Awad et al. (2018). They highlighted the critical involvement of chemerin, leptin and psoriasin in the pathogenesis of psoriasis as well as their eminent association with the disease severity. They reported that, the three biomarkers were implicated in various systemic inflammatory and immune-modulatory pathways that enhance the production of other pro-inflammatory cytokines, provoke the anti-apoptotic processes in T-cells and promote the proliferation of keratinocytes.

The current study revealed higher CIMT and EFT values in psoriatic patients than in controls in addition to positive correlation with PASI. Similar results were obtained by Asha et al. (2014) and Awad et al. (2018) who stated that; carotid intimal smooth muscle 
proliferation, overproduction of inflammatory mediators and endothelial dysfunction could explain the increased CIMT and premature atherosclerosis in psoriasis patients and its relation to disease severity. In addition, Bulbul et al. (2013) and Aksu et al. (2017) demonstrated higher EFT values in psoriasis patients and clarified that the activation of immune-mediated pathways together with the production of inflammatory adipokines such as leptin might be involved.

Our data showed that; CIMT in psoriatic patients was positively correlated with WHR, TC, TGs, LDL-c, hs-CRP, and inversely with HDL-c. Asha et al. (2014) and Wang et al. (2017) obtained matching results and declared that; increased CIMT in psoriasis has frequently associated with detectable clusters of atherogenic risk factors as dyslipedemia, abdominal adiposity and inflammatory markers. These clusters worked cooperatively, contributing to early-stage of endothelial dysfunction, therefore their linkage to CVD and atherosclerosis in psoriasis patients. Additionally, CIMT was positively correlated with plasma chemerin, leptin and psoriasin. These results were in consistence with Asha et al. (2014); Aksu et al. (2017) and Awad et al. (2018) who elucidated that chemerin, leptin and psoriasin being over-produced in psoriasis, could enhance carotid intimal smooth muscle proliferation, endothelial dysfunction and subsequently increased CIMT. Although the three markers can act independently, the presence of their positive correlations with CIMT might indicate their synergistic effect.

Our results, as well as Bulbul et al., 2013 and Aksu et al., 2017 showed that, EFT was positively correlated with WHR, TC, TGs, LDL-c, hs-CRP, chemerin, leptin and psoriasin and inversely with HDL-c in psoriatic patients. This could be interpreted as, EFT is an active metabolic tissue producing various atherogenic and inflammatory cytokines and participating in cellular infiltration and endothelial dysfunction of the vascular intima. Balta et al. (2016) reported positive correlations with dyslipidemia, WHR, immuneinflammatory markers, CIMT and increased cardiovascular risk in psoriasis, thus EFT might be a promising marker for risk stratification and premature atherosclerosis in psoriasis patients.

Multiple linear regression analysis of significant factors affecting CIMT and EFT showed that; plasma chemerin, psoriasin, hs-CRP and 
leptin were independently correlated with CIMT and displayed high significance for predicting its value, likewise plasma leptin, chemerin and psoriasin were independently correlated with EFT and demonstrated high significance for prediction of its value. Our data were in agreement with Asha et al. (2014); Aksu et al. (2017) and Awad et al. (2018) who stated that, the cumulative effects of chemerin, leptin and psoriasin on CIMT and EFT denoting the significant correlation of cardiovascular risk factors and inflammatory biomarkers. In addition, high CIMT and EFT together with elevated plasma chemerin, leptin and psoriasin may exhibit synergistic effect to increase the risk of subclinical atherosclerosis in psoriasis patients. In line with these findings, CIMT and EFT might be used as reliable diagnostic markers for predicting the future cardiovascular risks and being as an early sign of subclinical atherogenesis in patients with psoriasis.

Certain limitations in our study included; relatively small sample size and different protocols for measuring CIMT and EFT such as computed tomography (CT) and magnetic resonance imaging (MRI) that led to variability of the measurements within the studies.

\section{CONCLUSION}

Our data concluded that chemerin, leptin and psoriasin might be an important association between psoriasis and atherosclerosis. Screening for plasma chemerin, leptin and psoriasin along with CIMT and EFT as potential markers of subclinical atherosclerosis in psoriasis patients is suggested. CIMT and EFT are simple, non-invasive and reliable methods for predicting risk stratification and subclinical atherosclerosis in psoriasis patients in order to reduce their morbidity and mortality.

Acknowledgments: The authors are indebted and thankful to all patients, volunteers and paramedics for their assistance in the research.

Conflict of Interests \& Funding: The authors declared no competing interests with respect to authorship and publication of this article. This research was not funded from any organization. 


\section{REFERENCES}

Aksu F, Caliskan M, Keles N, Erek Toprak A, Uzuncakmak TK, Kostek O, Yilmaz Y, Demircioglu K and Cekin E (2017): Chemerin as a marker of subclinical cardiac involvement in psoriatic patients. Cardiol J; 24(3):276-283.

Asha K, Sharma SB, Singal A and Aggarwal A (2014): Association of carotid intima-media thickness with leptin and apoliprotein b/apoliprotein a-I ratio reveals imminent predictors of subclinical atherosclerosis in psoriasis patients. Acta Medica; 57(1):21-27.

Awad SM, Attallah DA, Salama RH, Mahran AM and Abu ElHamed E (2018): Serum levels of psoriasin (S100A7) and koebnerisin (S100A15) as potential markers of atherosclerosis in patients with psoriasis. Clin Exp Dermatol; 43(3):262-267.

Balta S, Ozturk $\mathbf{C}$ and Celik T (2016): The Relation between Epicardial Fat Thickness and Prognostic Risk Scores. Arquivos Brasileiros de Cardiologia; 107(6):607.

Banas M, Zegar A, Kwitniewski M, Zabieglo K, Marczynska J, Kapinska-Mrowiecka M, Zabel BA and Cichy J (2015): The expression and regulation of chemerin in the epidermis. PLoS ONE; 10 (2): e0117830.

Bulbul Sen B, Atci N, Rifaioglu EN, Ekiz O, Kartal I, Buyukkaya E, Kurt M, Buyukkaya S, Akcay AB and Sen N (2013): Increased epicardial fat tissue is a marker of subclinical atherosclerosis in patients with psoriasis. Br J Dermatol; 169(5):1081-1086.

Cetin M, Cakici M, Polat M, Suner A, Zencir C and Ardic I (2013): Relation of Epicardial Fat Thickness with Carotid IntimaMedia Thickness in Patients with Type 2 Diabetes Mellitus. International Journal of Endocrinology; 2013:769175.

Cunden LS, Brophy MB, Rodriguez GE, Flaxman HA and Nolan EM (2017): Biochemical and Functional Evaluation of the Intramolecular Disulfide Bonds in the Zinc-Chelating Antimicrobial Protein Human S100A7 (Psoriasin). Biochemistry; 56 (43):5726-5738.

Dattilo G, Imbalzano E, Casale M, Guarneri C, Borgia F and Mondello S (2018): Psoriasis and Cardiovascular Risk: Correlation Between Psoriasis and Cardiovascular Functional Indices. Angiology; 69(1):31-37. 
Dinić M, Zečević RD, Hajduković Z, Mijušković M, Đurić P, Jović Z, Grdinić A and Kandolf-Sekulović $L$ (2016): Psoriasis is the independent factor for early atherosclerosis: A prospective study of cardio-metabolic risk profile. Vojnosanit Pregl; 73 (12):1094-1101.

Eichelmann F, Weikert C, di Giuseppe R, Biemann R, Isermann B and Schulze MB (2017): Methodological utility of chemerin as a novel biomarker of immunity and metabolism. Endocr Connect; 6 (5):340-347.

Ekman AK, Vegfors J, Eding CB and Enerbäck C (2017): Overexpression of Psoriasin (S100A7) Contributes to Dysregulated Differentiation in Psoriasis. Acta Derm Venereol; 97(4):441-448.

El-Boghdady NA, Ismail MF, Abd-Alhameed MF, Ahmed AS and Ahmed HH (2018): Bidirectional Association Between Psoriasis and Obesity: Benefits and Risks. J Interferon Cytokine Res; 38(1):12-19.

Fossati $P$ and Prenciphe $L$ (1982): Determination of serum triglyceride. Clin Chem; 28: 207-210.

Friedewald W, Levy R and Fredrickson D (1972): Estimation of the concentration of low-density lipoprotein cholesterol in plasma without use of the preparative ultracentrifuge. Clin Chem; 18: 499502.

Furue M, Tsuji G, Chiba T and Kadono T (2017): Cardiovascular and Metabolic diseases comorbid with psoriasis: Beyond the skin. Intern Med; 56: 1613-1619.

Gordon T, Zidek W and Amer M (1977): Determination of highdensity lipoprotein cholesterol. J Med; 42: 707-710.

Hawkes JE, Chan TC and Krueger JG (2017): Psoriasis pathogenesis and the development of novel targeted immune therapies. J Allergy Clin. Immunol; 140: 645-653.

Janssen I (2013): The public health burden of obesity. Can J Diabetes; 37:90-96.

Kolliker Frers RA, Cosentino V and Tau J (2018): ImmuneMediated Inflammation Promotes Subclinical Atherosclerosis in Recent-Onset Psoriatic Arthritis Patients without Conventional Cardiovascular Risk Factors. Frontiers in Immunology; 9:139.

Kothiwala SK, Khanna N, Tandon N, Naik N, Sharma VK, Sharma S and Sreenivas V (2016): Prevalence of metabolic syndrome and cardiovascular changes in patients with chronic plaque 
psoriasis and their correlation with disease severity: A hospital-based cross-sectional study. Indian J Dermatol Venereol Leprol; 82 (5):510518.

Kyriakou A, Patsatsi A, Sotiriadis D and Goulis DG (2017): Serum Leptin, Resistin, and Adiponectin Concentrations in Psoriasis: A Meta-Analysis of Observational Studies. Dermatology; 233(5):378389.

Michalek IM, Loring B and John SM (2017): A systematic review of worldwide epidemiology of psoriasis. J Eur Acad Dermatol Venereol; 31:205-212.

Rashiti P, Behluli I and Bytyqi AR (2017): Assessment of the Correlation between Severity of Coronary Artery Disease and WaistHip Ratio. Open Access Maced J Med Sci; 5(7):929-933.

Rifai $\mathbf{N}$ and Warnick $\mathbf{R}$ (2006): Lipids, lipoproteins, apolipoproteins and other cardiovascular risk factors: Tietz Textbook of Clinical Chemistry and Molecular Diagnosis. Carl, A. B, Edward, R. A and David, E. B [edrs.]. Saunders. [4th edition].Ch 26.PP. 918-922.

Santilli S, Kast DR, Grozdev I, et al. (2016): Visualization of atherosclerosis as detected by coronary artery calcium and CIMT reveals significant atherosclerosis in a cross-sectional study of psoriasis patients in a tertiary care center. Journal of Translational Medicine; 14(1):217.

Trinder P (1969): Determination of glucose in blood using glucose oxidase with an alternative oxygen acceptor. J Ann Clin Biochem; 6: 24-25.

Wang X, Dalmeijer GW, den Ruijter HM, et al. (2017): Clustering of cardiovascular risk factors and carotid intima-media thickness: The USE-IMT study. PLoS ONE; 12(3):e0173393.

Wang X, Guo Z, Zhu Z, Bao Y and Yang B (2016): Epicardial fat tissue in patients with psoriasis: a systematic review and metaanalysis. Lipids Health Dis; 15:103.

Yang JS, Gerber JN and You HJ (2017): Association between fasting insulin and high-sensitivity $\mathrm{C}$ reactive protein in Korean adults. BMJ Open Sport Exercise Medicine; 3 (1):e000236.

Zhang Y and Chua S (2017): Leptin Function and Regulation. Compr Physiol; 8 (1):351-369. 


\section{الملخـــص العــــي}

\section{مستوى الكيميرين والليتين والسورياسين في البلازما كدلالات محتملة لتصلب الثرايين ما قبل الاكلينيكي في مرضي الصين الصدفية}

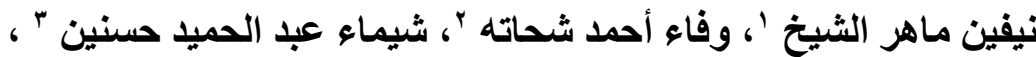

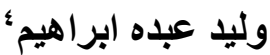

\section{أقسام: ' الكيمياء الحيوية الطبية و البيولوجيا الجزيئية، r الجلاية والتناسلية

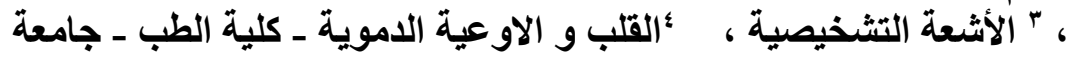 المنوفية.}

يعتبر الكيميرين و الليتين والسورياسين من البروتينات المحفزة للالتهابات والمعدلة للمناعة وتصاحب مرض الصدفية حيث ترتفع مستوياتها. وقد تم دراسة علاقة هذه البروتينات

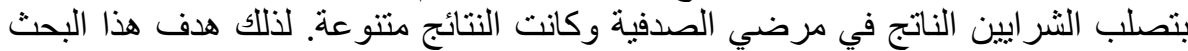

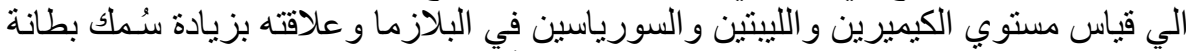

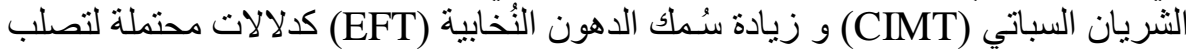

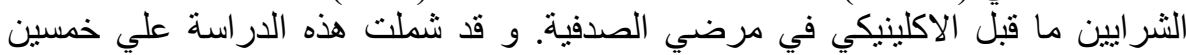

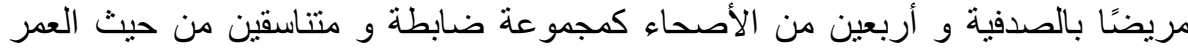

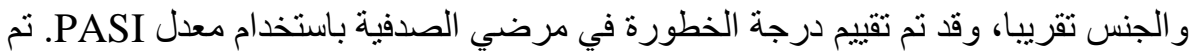

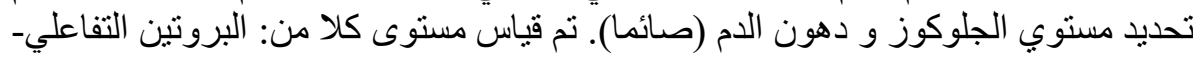

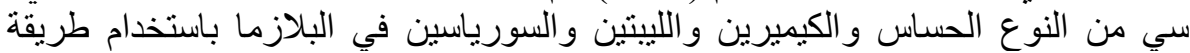

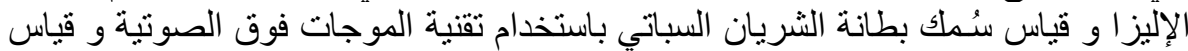

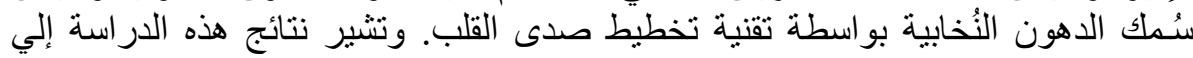

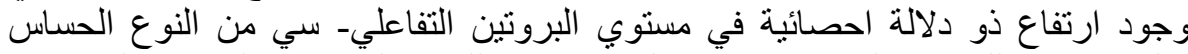

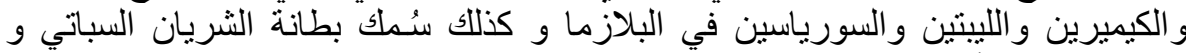

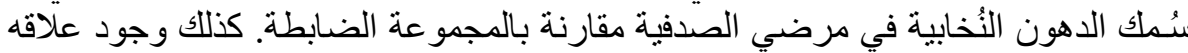

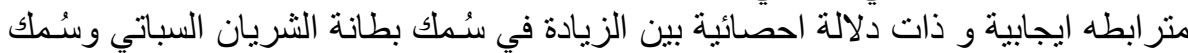

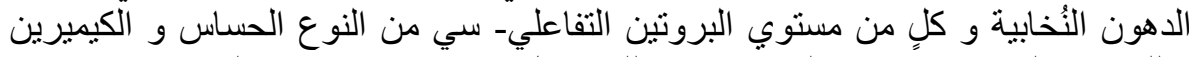

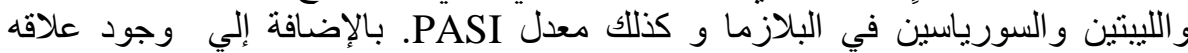
متر ابطه ايجابية و ذات دلالة احصائية بين معدل PASI و كلٍ من مستوي البي البروتين

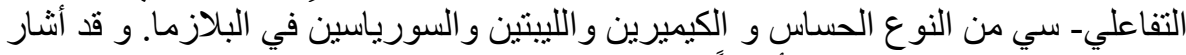

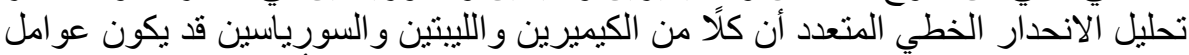

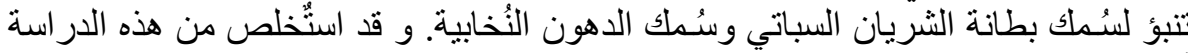

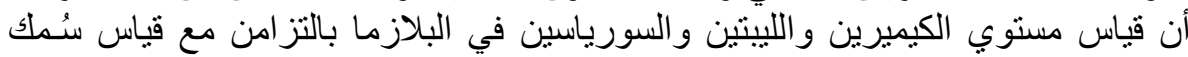


Nevein M. Al-sheikh et all

بطانة الثريان السباتي وسُمك الدهون النُخابية قد تكون عوامل ذات قيمة تنبؤية لتصلب الثر ايين ما قبل الاكلينيكي في مرضي الصئي الصدية الصنية. 